\title{
Computed tomography imaging of septic shock. Beyond the cause: the "CT hypoperfusion complex". A pictorial essay
}

\author{
Marco Di Serafino ${ }^{1 *+}$ (D) Daniela Viscardi ${ }^{2 \dagger}$, Francesca lacobellis ${ }^{1 \dagger}$, Luigi Giugliano ${ }^{3}$, Luigi Barbuto ${ }^{1}$, \\ Gaspare Oliva', Roberto Ronza' , Antonio Borzelli ${ }^{4}$, Antonio Raucci ${ }^{1}$, Filomena Pezzullo ${ }^{1}$, \\ Maria Giovanna De Cristofaro ${ }^{2}$ and Luigia Romano ${ }^{1}$
}

\begin{abstract}
Septic shock is a medical emergency that represents one of the most important underlying causes for presentation to the Emergency Department. Sepsis is defined as organ dysfunction, life-threatening event caused by a deregulated inflammatory host response to infection, with a mortality risk ranging from 10 to $40 \%$. Early sepsis identification is the cornerstone of management and diagnostic imaging can play a pivotal role in this clinical context. The choice of imaging modality depends on several factors, associated with the clinical condition and the presence or absence of localising signs and symptoms. The diagnostic accuracy of contrast-enhanced total-body CT has been well established during septic shock, allowing for a rapid, panoramic, and detailed study of multiple body areas, simultaneously. The aim of this article is to illustrate the controversial CT hypoperfusion complex in patients with septic shock, characterised by the following imaging features: decreased enhancement of the viscera; increased mucosal enhancement; luminal dilation of the small bowel; mural thickening and fluid-filled loops of the small bowel; the halo sign and flattening of the inferior vena cava; reduced aortic diameter; peripancreatic oedema; abnormal parenchymal perfusion; and other controversial findings that are variably associated with each other and reversible during the early stages. Increasing physicians' awareness of the significance of these findings could prompt alternative approaches to the early assessment and management of septic shock. In this perspective, CT imaging represents a useful tool for a complete, rapid and detailed diagnosis of clinically suspected septic shock, which can be used to improve patient outcomes.
\end{abstract}

Keywords: Sepsis, Septic shock, Diagnostic imaging, Contrast-enhanced CT, CT hypoperfusion complex

\section{Key points}

- Sepsis is a life-threatening event with a mortality risk ranging from 10 to $40 \%$.

- Early patient recognition is a cornerstone of sepsis management.

\footnotetext{
*Correspondence: marco.diserafino@aocardarelli.it

${ }^{\dagger}$ Marco Di Serafino, Daniela Viscardi and Francesca lacobellis contributed equally to this work and they are co-first authors

${ }^{1}$ Department of General and Emergency Radiology, "Antonio Cardarelli"

Hospital, Antonio Cardarelli st 9, 80131 Naples, Italy

Full list of author information is available at the end of the article
}

- Total-body CT imaging plays a pivotal role in septic shock condition.

- CT hypoperfusion complex recognition may facilitate early diagnosis improving treatment planning in septic shock patients.

\section{Introduction}

Septic shock qualifies as a medical emergency and represents one of the most important causes for presentation to the Emergency Department (ED). Sepsis refers to life-threatening organ dysfunction caused by a 
deregulated host response to infection [1-4]. According to the new Septic 2016 definition, also referred to as Sepsis-3, septic shock patients can be identified by the co-occurrence of sepsis symptoms with persistent hypotension that requires vasopressors to maintain a mean arterial pressure of $65 \mathrm{mmHg}$ or higher or serum lactate levels $>2 \mathrm{mmoL} / \mathrm{L}(18 \mathrm{mg} / \mathrm{dL})$, despite adequate volume resuscitation $[1,4]$. This revised and current definition emphasises that sepsis is the primary cause of death from infection [1-4]. Sepsis-associated mortality is high, ranging from 10 to $40 \%$, and the incidence of sepsis has continuously increased in parallel with the average age of the population and the increased invasiveness of diagnostic and therapeutic procedures [4]. Mortality is associated with multiple pathogenic factors, including the timeliness of diagnosis and the execution of appropriate and early treatment [1-4]. Due to the high mortality rate, the early identification and appropriate management of sepsis are crucial to improving outcomes as well as timedependent emergencies such as polytrauma, acute myocardial infarction, or stroke. The management of septic shock requires prompt recognition and the appropriate administration of antibiotic therapy, haemodynamic support, and the identification and treatment of the infection source [5,6]. Early sepsis identification is the cornerstone of management, and diagnostic imaging can play a pivotal role in this clinical context. A wide range of imaging tools is currently available for the investigation of septic shock. The choice of imaging modality depends on several factors associated with the clinical condition and the presence or absence of signs and symptoms that can be used to localise the source of sepsis. The diagnostic accuracy of total-body computed tomography $(\mathrm{CT})$ has been well established for the identification of septic shock, allowing for a rapid and simultaneous study of multiple body areas, generating detailed and panoramic images. The aim of this article is to review the characteristics of septic shock from an imaging perspective, beyond the underlying causes and to highlight how CT can be used to identify a variety of septic shock-related signs that are collectively described as CT hypoperfusion complex. The latter describes a set of widely reported signs and symptoms that are commonly observed during traumaassociated hypovolaemic shock and can be used to identify septic shock. The early recognition, diagnosis, and treatment of septic shock have profound prognostic and therapeutic implications.

\section{Etiopathogenesis}

The factors that contribute to septic shock occurrence are associated with an extremely varied range of infections pathologies that can involve any area of the body. Most cases of septic shock are caused by gram-negative bacilli
(Escherichia coli, Klebsiella pneumoniae, Enterobacter spp., Acinetobacter baumannii, and Pseudomonas aeruginosa) or nosocomial gram-positive cocci (Staphylococcus aureus and coagulase-negative Staphylococcus) cocci, and septic shock most often occurs in immunocompromised patients and in those with chronic and debilitating diseases. Antimicrobial-resistant bacteria, such as methicillin-resistant Staphylococcus aureus and vancomycin-resistant Enterococci, are often detected in septic patients with nosocomial infections. Invasive candidiasis and other uncommon pathogens should be considered in particular conditions, such as in neutropenic patients [7, 8]. The most common sites of infection include the lungs (pneumonia, empyema and lung abscess), urinary tract (obstructive urosepsis), biliary tract (cholangitis), gallbladder (acute cholecystitis), gastrointestinal tract (acute appendicitis and colic abscesses), skin/soft tissue, intravascular catheters, central nervous system, and endocardium. Subtle signs may exist, such as septic thrombosis of vascular access, mycotic aneurysms, abscess formations, and Fournier's gangrene [3-7]. The pathogenesis of septic shock is complex, and the roles played by the inflammatory process and coagulation are highly intricate. During the initial phase, a transient dilation of the arteries and arterioles occurs, accompanied by a reduction in peripheral arterial resistance, associated with a characteristic increase in cardiac output. This pathophysiological stage has been termed "hot shock" or "high-range shock". Subsequently, cardiac output decreases, blood pressure decreases (with or without an increase in peripheral resistance), and the typical aspects of shock appear, resulting in reduced perfusion. This cascade of responses results in the dysfunction of one or more organs, inducing disseminated intravascular coagulation (DIC) and causing death $[1,8,9]$.

\section{Signs and symptoms}

The signs and symptoms of sepsis are highly variable and clinical diagnosis often anticipates the culture results [10]. Typical signs and symptoms of sepsis include: high fever $\left(>38{ }^{\circ} \mathrm{C}\right)$, chills, diaphoresis, tachycardia, increased respiratory rate, substantial reduction of diuresis, confusion, oedema, and impairment of the general state, in addition to symptoms related to the infection. Headaches, rash, bruising, or bleeding are also common [1-4]. If left untreated, septic shock can progress to hypotension refractory to treatment, with paradoxically hot skin, oliguria, lactic acidosis, sensory alterations, and signs of impairment associated with at least one organ associated with the basic septic process. Care should be taken because sepsis can also present with nuanced manifestations that 
can be mistaken for other disorders, such as delirium, heart failure, and pulmonary embolism (Table 1) [1-4].

\section{Diagnosis}

The diagnosis is clinical and considers various parameters, including blood pressure, heart rate and $\mathrm{O}_{2}$ monitoring, blood count with leukocyte formula, serum electrolytes, creatinine, lactates, the invasive monitoring of central venous pressure, $\mathrm{PaO}_{2}$, central venous $\mathrm{O}_{2}$ saturation, blood cultures, urine, and the monitoring of other potential sites of infection, especially wounds in surgical patients. Sepsis is suspected when a patient with a known infection develops systemic signs of inflammation or organ dysfunction, which requires the avoidance of septic shock and the exclusion of other potential causes for shock (e.g. hypovolemia, myocardial infarction). No gold standard diagnostic test currently exists for the identification of septic shock. However, a new bedside index, called quick Sequential Organ Failure Assessment (qSOFA) score, can be used to identify patients with suspected infection who are being treated outside of critical care units and are likely to have a prolonged intensive care unit (ICU) stay or to die in the hospital. The qSOFA requires at least 2 of following 3 risk variables: respiratory rate of 22 or more breaths per minute, systolic blood pressure of $100 \mathrm{mmHg}$ or less, and altered mental status. The qSOFA does not require laboratory tests and can be assessed easily and repeatedly (Table 2) [1, 11]. Sepsis is associated with vasodilation, capillary leak, and decreased effective circulating blood volume, reducing venous return. These haemodynamic effects result in impaired tissue perfusion and organ dysfunction. Although localised signs and symptoms of organ dysfunction may be present, organ hypoperfusion or shock can manifest without knowledge of causation [10]. The goals of resuscitation in cases of sepsis and septic shock include the restoration of intravascular volume, increased oxygen delivery to tissues, and the reversal of organ dysfunction [6]. Although fluid administration will significantly increase cardiac output, the goals should be individualised for each patient, according to the evaluated need for fluids and each patient's premorbid conditions [1, 12]. A fundamental recommendation is to initiate the necessary diagnostic process to identify the infection source while prioritising the management of haemodynamics and vital functions. The early identification of infection source and the rapid remediation of infection are essential for the appropriate management of the septic patient $[1,12]$.

\section{Diagnostic imaging}

The importance of imaging for the establishment of the infection source is well recognised [1]. A wide range of imaging tools is currently available for the investigation of septic shock. The choice of imaging modality depends on several factors, the most important of which include the overall clinical condition of the patient and the presence or absence of localising signs and symptoms as summarised in Table 1 [12-21].

The diagnostic accuracy of CT for the identification of the source of clinically suspected septic shock has been well established, allowing for the rapid and detailed study of multiple body areas simultaneously. CT provides a standardised method for patient evaluation, which is not operator-dependent. In a few minutes, CT can provide images of multiple body regions, simultaneously, improving the ability to identify the septic source, which can improve patient management $[1,22]$. CT also played an increasingly important role in the guidance of aspiration and drainage, with a high degree of accuracy [16]. CT should be performed in all patients with a spiral technique, craniocaudal acquisition and in a supine position with abducted upper limbs, to reduce the radiation dose and guarantee a higher image quality of the thoraco-abdominal organs. Breath-hold acquisitions can ensure the avoidance of motion artefacts [22]. In emergency settings, CT examinations should include both unenhanced and contrast-enhanced acquisitions of the abdomen. High concentrations (370-400 mg I/ $\mathrm{mL}$ ) of intravenous (IV) contrast medium $(80-130 \mathrm{~mL}$ iodinated contrast medium, depending on the patient's weight), injected at $3.5-4 \mathrm{~mL} / \mathrm{s}$ through an 18 -gauge needle into the antecubital vein should be administered, followed by a bolus of $40 \mathrm{~mL}$ saline at the same flow rate. The acquisition of the arterial phase is timed with the bolus tracking by placing the region of interest (ROI) on the aortic arch and starting at an attenuation threshold of 100 Hounsfield Unit (HU). The portal-venous phase is acquired with a delay of 60-70 s after the beginning of the injection. The suggested acquisition volume in emergency settings includes the abdomen and pelvis scan in arterial phase and the chest, abdomen, and pelvis in portal phase, to obtain a complete examination. An additional, late scan of the abdomen and pelvis at 3-5 min may also be acquired to address various causes of abdominal pain. The rectal administration of contrast material is not typically useful. For adequate analysis and postprocessing, with maximum intensity projection (MIP) and multiplanar reformation (MPR), an effective slice thickness of $2.5 \mathrm{~mm}$, with reconstruction at $0.625 \mathrm{~mm}$, is recommended. Automatic tube current modulation should be adopted to reduce radiation exposure, and the standard reconstruction algorithm should be applied. 


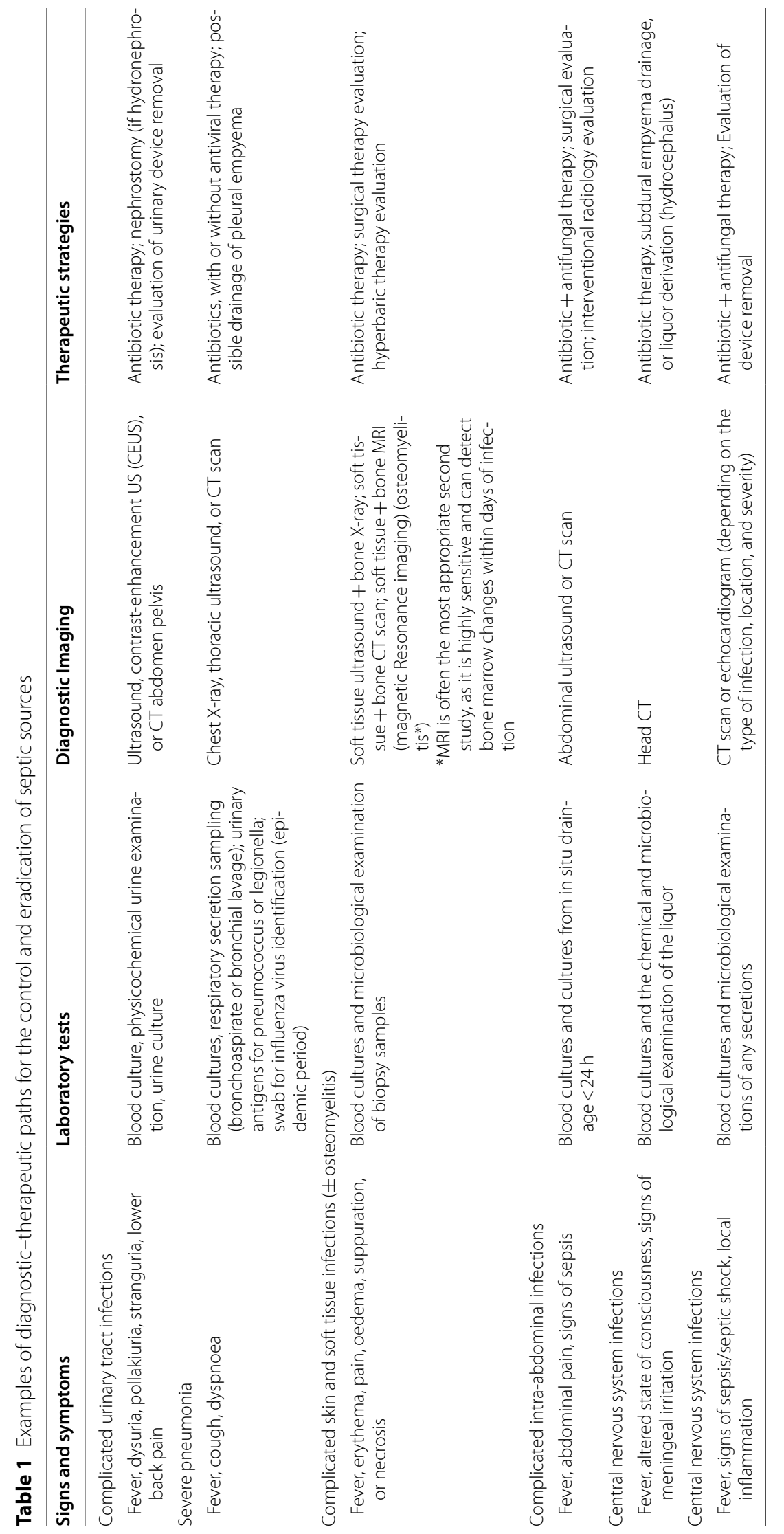


Table 2 Quick sequential organ failure assessment (qSOFA) score

Quick sequential organ failure assessment (qSOFA)

Respiratory rate $\geq 22 /$ min

Change in mental status

Systolic blood pressure $\leq 100 \mathrm{mmHg}$

Head CT should be considered for patients presenting with altered mental status, without IV contrast medium administration; head CT should be performed before the total body study to exclude the presence of intracranial haemorrhage. Head CT can also be performed after the late phase of the total body study to exclude intracranial abscess or malignancy [22].

\section{CT hypoperfusion complex}

In addition to being used to identify the underlying cause of the septic state, CT is required for the early recognition of shock-associated CT imaging signs, collectively referred to as CT hypoperfusion complex, which can improve patient prognosis and management. The CT hypoperfusion complex is frequently associated with hypotension, which can also present in many no sepsis related clinical conditions, such as trauma-induced hypotensive shock (e.g. severe head or spine injury), cardiac arrest, and diabetic ketoacidosis [23-26]. The CT hypoperfusion complex has important prognostic and therapeutic implications and must be promptly recognised. However, although the pathogenic mechanisms that underlie hypotensive shock and septic shock are quite different, the CT findings associated with these two syndromes are often comparable to those that have been widely described in previous literature as in posttraumatic hypotensive shock, which can be grouped into vascular, visceral, and parenchymal signs. These signs include the decreased enhancement of the viscera, the increased mucosal enhancement and luminal dilation of the small bowel, the mural thickening and identification of fluid-filled loops in the small bowel, the halo sign and flattening of the inferior vena cava (IVC), reduced aortic diameter, peripancreatic oedema and other controversial parenchymal and visceral findings and ascites that can occur in varying combinations and are often and reversible during early stages. The presence of 2 or more vascular, visceral, or parenchymal signs is necessary to establish the presence of CT hypoperfusion complex [20-23] (Table 3).

Vascular signs include diminished inferior vena cava diameter, diminished aortic diameter, and abnormal vascular enhancement.

\section{Flattening of the inferior vena cava}

The flattening of the IVC calibre has been defined as the identification of reduced anterior-posterior diameter $(<9 \mathrm{~mm})$ in three consecutive segments, $20 \mathrm{~mm}$ above and below the renal veins, and at the level of the perihepatic portion (Fig. 1). Flattening is the result of decreased circulating blood volume and indicates reduced venous return in patients with systemic hypotension, which may not be appreciable due to the massive infusion of liquids. In addition, variations in intra-abdominal pressure and the respiratory cycle can also affect the IVC diameter. IVC flattening has a specificity of $90 \%$ and a sensitivity of $84 \%$ for the identification of hypoperfusion shock due to sepsis in spontaneously breathing patients, whereas the sensitivity and specificity are both $90 \%$ in ventilated patients [20-23].

\section{The halo sign}

The presence of low-density fluid $(<20 \mathrm{HU})$ surrounding the IVC, known as an IVC halo, can be identified in approximately $80 \%$ of patients with severe hypotension (Fig. 2a). The IVC halo represents the presence of extracellular fluid, which may be due to a hyperpermeable state, secondary to systemic inflammatory response syndrome [23]. The halo sign is most frequently observed at the confluence of the hepatic veins into the IVC. The halo sign is not specific to hypoperfusive shock due to sepsis and can also be observed in other clinical settings, such as liver cirrhosis, congestion, or hepatitis [20-23].

\section{Small-calibre aorta}

A small-calibre abdominal aorta is defined as a reduced anteroposterior diameter $(<13 \mathrm{~mm})$ detected $20 \mathrm{~mm}$ above and below the renal arteries (Fig. 3). Small-calibre aorta occurs in approximately $30 \%$ of patients with systemic hypotension and is not specific to hypoperfusive shock due to sepsis, as it may be observed in the normal population. This sign is associated with vasoconstriction induced by the adrenergic system to compensate for the shock condition [23-27].

Visceral signs include bowel hypoperfusion also called shock bowel and mucosal enhancement of the gallbladder. 
Table 3 CT hypotension complex findings and frequency

\begin{tabular}{|c|c|c|c|}
\hline \multicolumn{4}{|c|}{ CT hypoperfusion complex } \\
\hline Type & Sign & Definition & $\begin{array}{l}\text { Incidence rate in } \\
\text { patient with severe } \\
\text { hypoperfusion* }\end{array}$ \\
\hline \multirow[t]{3}{*}{ Vascular signs } & Flattening of the inferior vena cava & $\begin{array}{l}\text { IVC flattening with anterior-posterior diameter < } \\
9 \mathrm{~mm} \text { in three consecutive segments, } 20 \mathrm{~mm} \\
\text { above and below the renal veins and at the level } \\
\text { of the perihepatic portion }\end{array}$ & $77-100 \%$ \\
\hline & The halo sign & $\begin{array}{l}\text { The presence of low-density fluid }<20 \mathrm{HU} \text { sur- } \\
\text { rounding the IVC }\end{array}$ & $77.8 \%$ \\
\hline & Small calibre aorta & $\begin{array}{l}\text { A small-calibre abdominal aorta with anterior-pos- } \\
\text { terior diameter }<13 \mathrm{~mm} \text { detected } 20 \mathrm{~mm} \text { above } \\
\text { and below the renal arteries }\end{array}$ & $20-48 \%$ \\
\hline \multirow[t]{2}{*}{ Visceral sign } & Shock bowel & $\begin{array}{l}\text { Small bowel fluid-filled dilated loops with thick- } \\
\text { ened walls (>3 mm) due to oedema of the } \\
\text { submucosa and increased mucosal enhance- } \\
\text { ment relative to the psoas muscle }\end{array}$ & $40-70 \%$ \\
\hline & Mucosal enhancement of the gallbladder & $\begin{array}{l}\text { Mucosal enhancement, without thickened gall- } \\
\text { bladder walls }\end{array}$ & $33.3 \%$ \\
\hline \multirow[t]{6}{*}{ Parenchymal signs } & $\begin{array}{l}\text { Peripancreatic oedema and abnormal pancreatic } \\
\text { enhancement (shock pancreas) }\end{array}$ & $\begin{array}{l}\text { Abnormal post-contrast higher attenuation than } \\
\text { to normal density values ( } 20 \mathrm{HU} \text { greater than the } \\
\text { liver and spleen) often with peripancreatic low- } \\
\text { density fluid }(<20 \mathrm{HU})\end{array}$ & $>44 \%$ \\
\hline & Splenic hypoperfusion & $\begin{array}{l}\text { Extremely decreased enhancement in contrast } \\
\text { early CT phase }\end{array}$ & $11-29.6 \%$ \\
\hline & Abnormal liver enhancement & $\begin{array}{l}\text { A reduction of hepatic enhancement ( } 25 \mathrm{HU} \text { less } \\
\text { than the spleen) }\end{array}$ & $4-11.1 \%$ \\
\hline & Abnormal renal enhancement & $\begin{array}{l}\text { Increased and prolonged parenchymal enhance- } \\
\text { ment }\end{array}$ & $55.6 \%$ \\
\hline & $\begin{array}{l}\text { Abnormal adrenal hyperenhancement (adrenal } \\
\text { stress) }\end{array}$ & Bilateral hyperenhancement of the adrenal gland & $>60 \%$ \\
\hline & Abnormal thyroid enhancement (shock thyroid) & $\begin{array}{l}\text { Heterogeneous contrast hyperenhancement, } \\
\text { similar to a multinodular gland, with the pres- } \\
\text { ence of low-density fluid surrounding the thyroid } \\
(5-10 \mathrm{HU})\end{array}$ & Not detected \\
\hline Other sign & Ascites & Fluid collects in peritoneal spaces & Not detected \\
\hline
\end{tabular}

*Literature data from post-traumatic hypoperfusion complex [26]

\section{Bowel hypoperfusion (shock bowel)}

The most frequent findings associated with shock bowel include fluid-filled, dilated loops with thickened walls $(>3 \mathrm{~mm}$ ) due to oedema of the submucosa and increased mucosal enhancement relative to the psoas muscle (Figs. 2b, 4a). Changes to the small intestine are the most commonly observed characteristics among the CT signs indicative of shock. The small intestine is often diffusely involved in the occurrence of hypotensive shock, whereas the colon is rarely involved [23-26]. The shock bowel symptoms occur due to systemic hypotension, with consequent sympathetic stimulation, resulting in splanchnic vasoconstriction and a reduction in intestinal perfusion. These effects reduce the supply of oxygen to the tissues, altering permeability and causing the hyperenhancement of the mucosa and the oedematous thickening of the intestinal wall and submucosa. The reduced reabsorption of fluids causes the luminal distension of the intestinal loops. The recognition of the shock intestine is essential to avoid confusion with other conditions, such as intestinal ischaemia due to vascular occlusion, which are associated with different CT characteristics. The most challenging differential diagnosis is diffuse bowel ischaemia due to vascular occlusion, which can also present with bowel-wall thickening and luminal distension. Unlike the shock bowel, bowel ischaemia due to arterial occlusion is not associated with the hyperenhancement of the mucosa or the congestion of the wall. Mesenteric venous occlusion may show both of these CT signs, however, in combination with a filling defect of the superior mesenteric vein or its branches, mesenteric congestion, and stranding [23]. In addition, CT signs associated with hypotension complex can be important indicators of systemic hypotension, which may facilitate the differential diagnosis. Shock bowel has a mortality rate of up to $70 \%$ [23-26, 28-31]. 


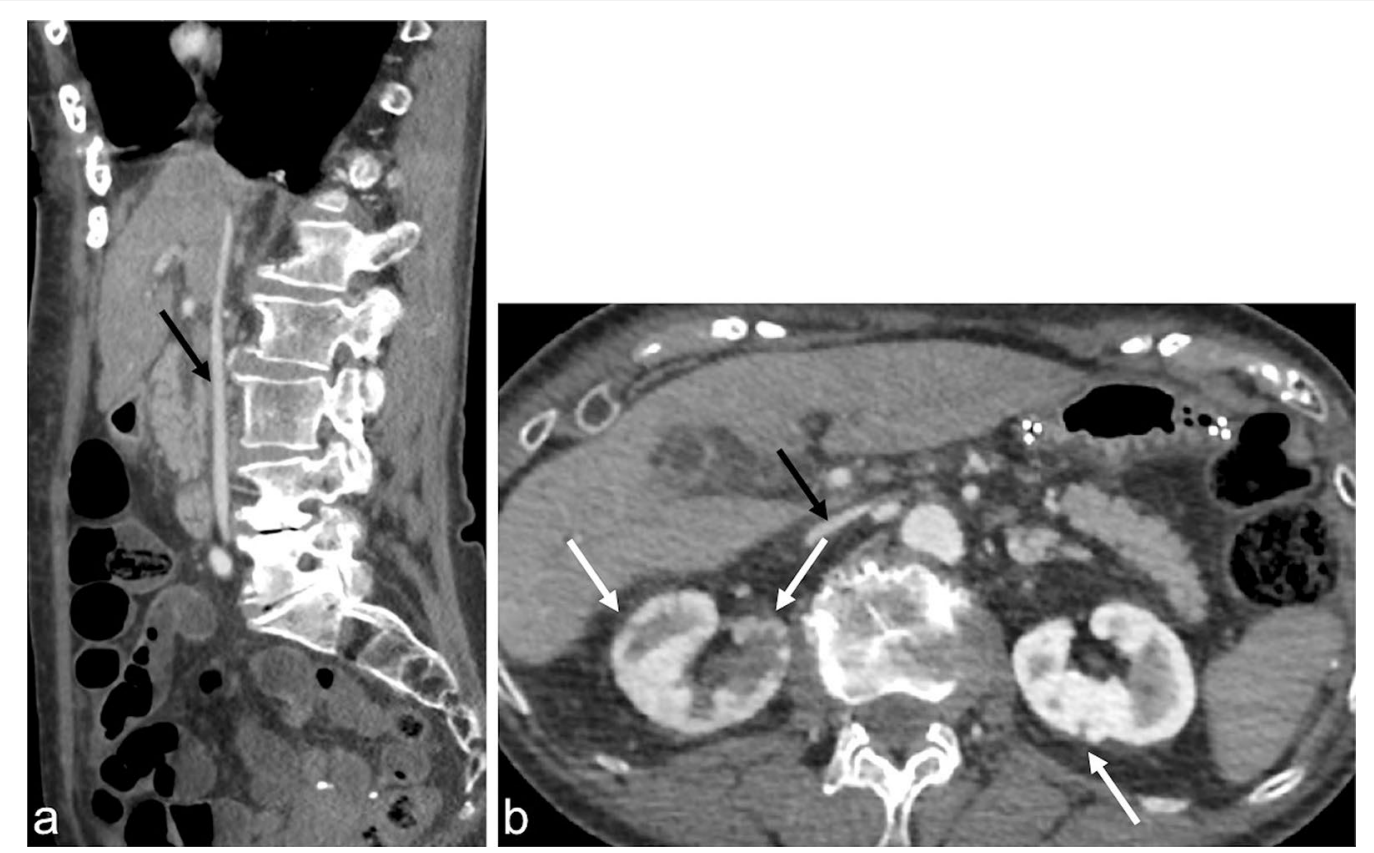

Fig. 1 Contrast-enhanced CT in the portal venous phase, showing an 84-year-old male with sepsis (qSOFA 2) due to bilateral nephritis (white arrows). A collapsed IVC can be observed in both the sagittal (a, black arrow) and axial views (b, black arrow)
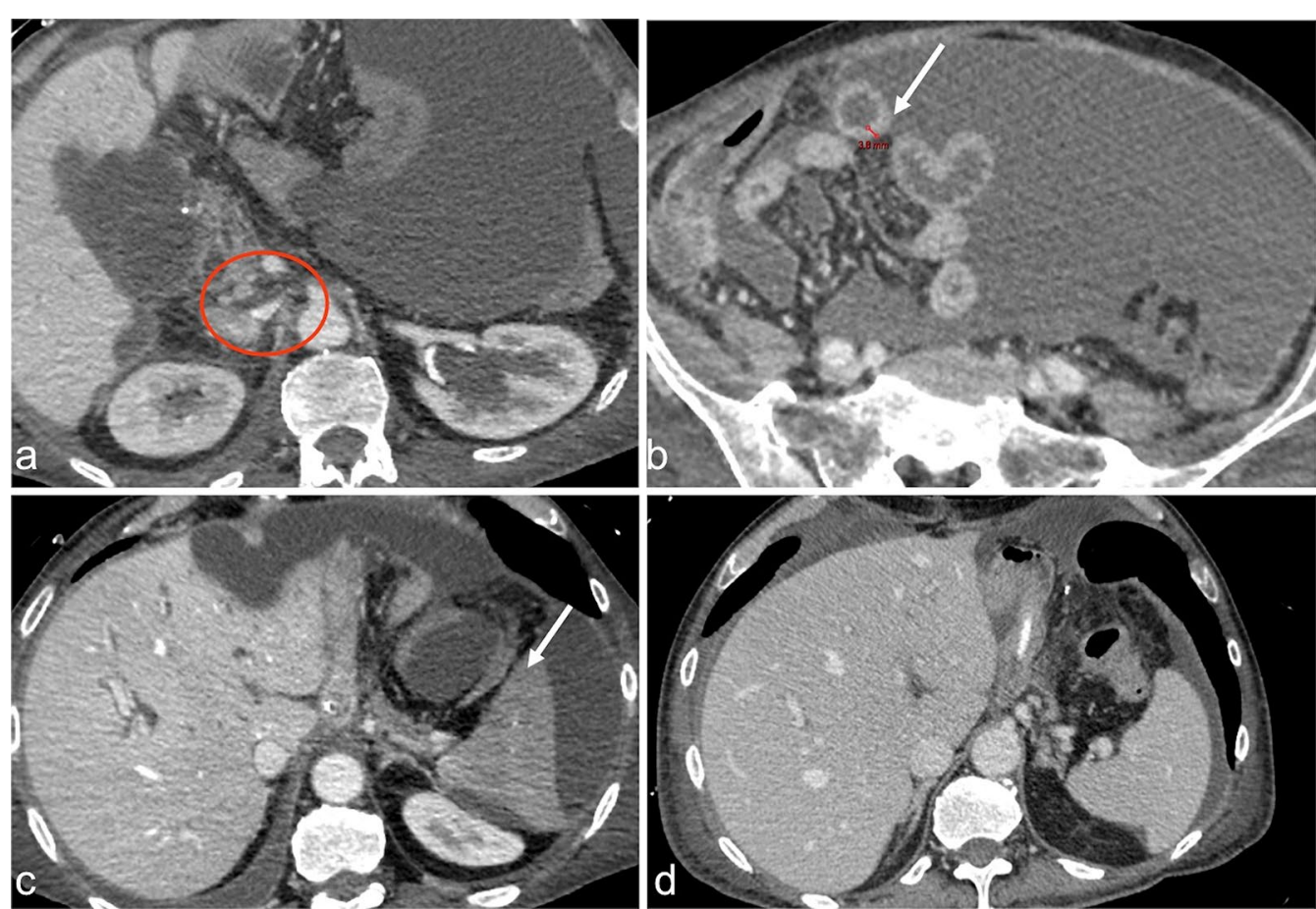

Fig. 2 Contrast-enhanced CT in the portal venous phase, in the axial view, showing a 70-year-old male with sepsis due to gastric cancer surgery complications (qSOFA 3). In this patient, multiple CT signs of sepsis can be observed, including inferior vena cava halo sign (a, red circle), thickened bowel wall (b, white arrow), reduced enhancement, and spleen volume (c, white arrow). Figure d shows a previous CT examination of the same patient for comparison 

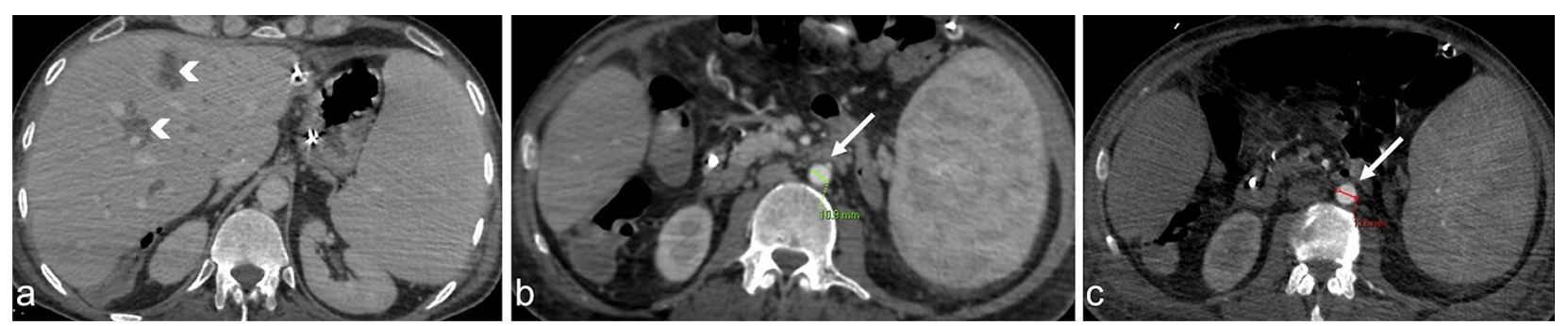

Fig. 3 Contrast-enhanced CT in the portal venous phase, in the axial plane, showing a 44-year-old female with sepsis (qSOFA 2) due to cholangitis with liver abscesses (a, arrowheads). The CT revealed the reduced calliper of the abdominal aorta (b, arrow), compared with the normal calliper of the abdominal aorta in a previous examination from the same patient (c)
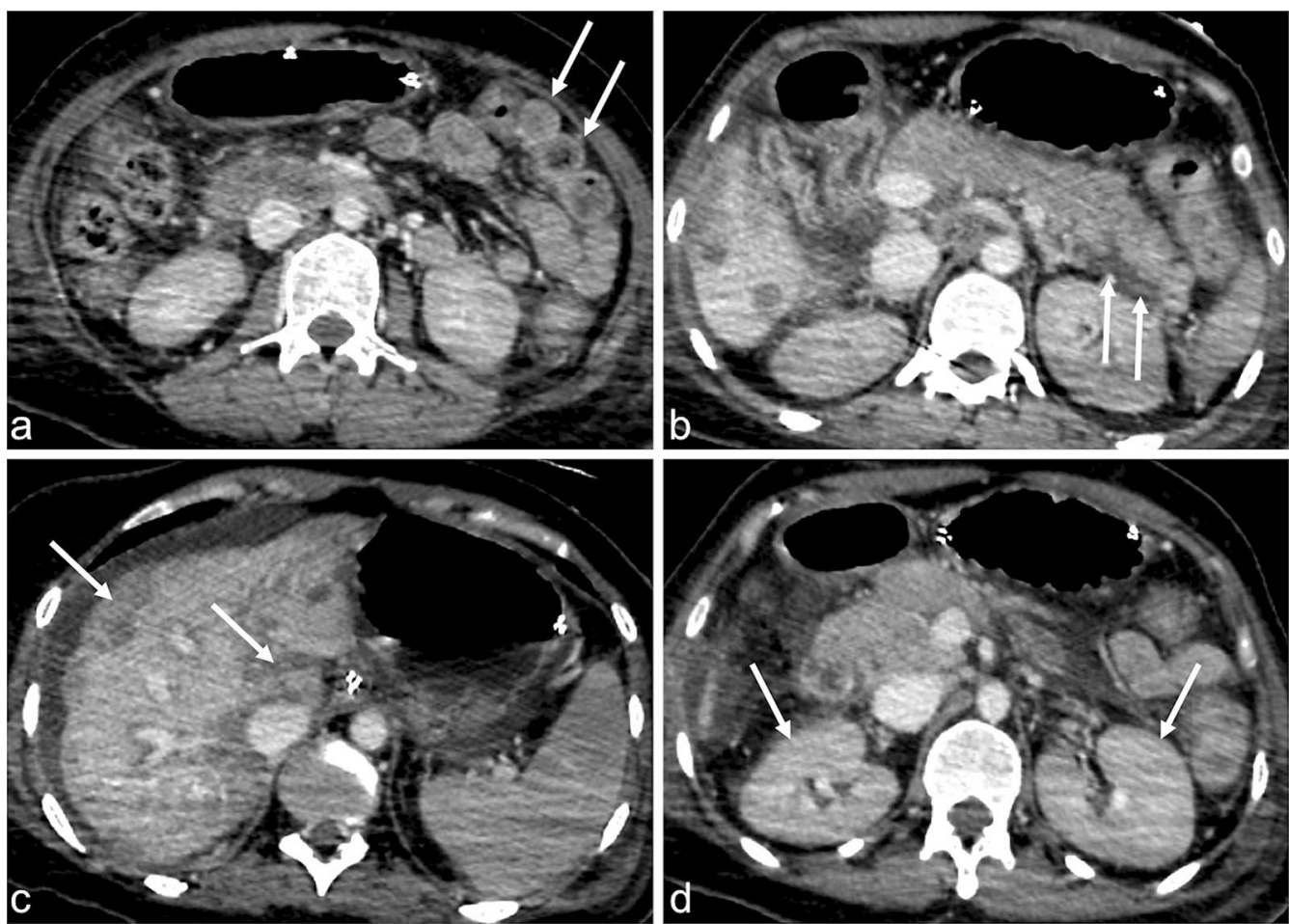

Fig. 4 Contrast-enhanced CT images in the portal venous phase, in the axial view, showing a 46-year-old female with lymphoma and sepsis due to infection (qSOFA 2). A thickened and hyperdense bowel wall can be observed (a, arrows), with peripancreatic oedema (b, arrows), reduced and inhomogeneous liver enhancement (c), and enlarged kidneys with abnormal enhancement (d, arrows)

\section{Mucosal enhancement of the gallbladder}

Mucosal enhancement, without thickened gallbladder walls, can be observed in hypotensive shock complex, with low specificity (Fig. 5) [23].

Parenchymal signs include abnormal pancreatic enhancement, associated with peripancreatic fluid, the hypoperfusion of the spleen and liver and the abnormal perfusion of the adrenal glands, kidneys and thyroid.

\section{Peripancreatic oedema and abnormal pancreatic} enhancement (shock pancreas)

Shock pancreas appears as an abnormal, post-contrast attenuation, with higher than normal density values $(20$ $\mathrm{HU}$ greater than the liver and spleen) and the presence of peripancreatic low-density fluid $(<20 \mathrm{HU})$, often in combination with mesenteric and other retroperitoneal fluid collections, at an incidence rate of up to $44 \%$ (Figs. 4b and 6). This phenomenon may be the result of cytokine 

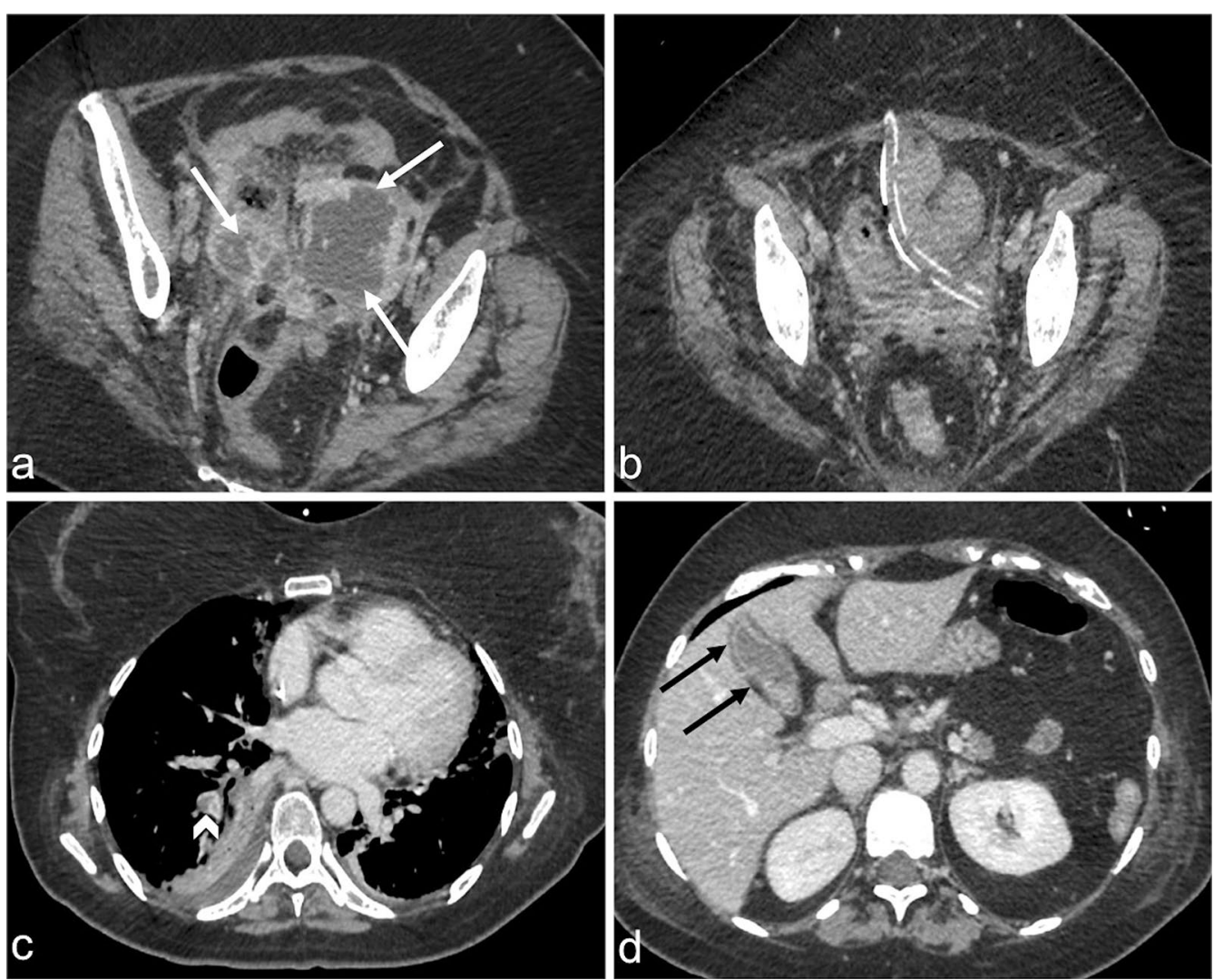

Fig. 5 Contrast-enhanced CT image in the portal venous phase showing a 44-year-old female with abscessed uterine neoplasm (a, arrows) and post-operative control with completely drained collection (b). Pulmonary thromboembolism (c, arrowhead) with development of a clinical septic state (qSOFA 2). Note the dense, gallbladder mural enhancement without thickened walls (d, black arrows)
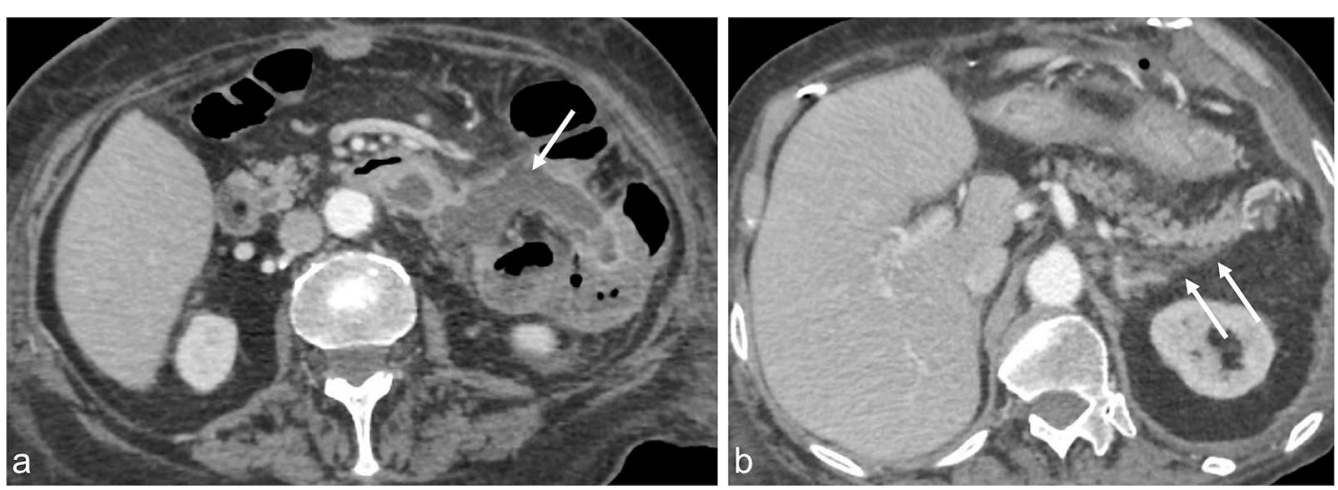

Fig. 6 Contrast-enhanced CT images in the portal venous phase, in the axial view, shows a 78-year-old female with sepsis (qSOFA 2) due to bowel anastomotic dehiscence (a, the white arrow indicates fluid collection caused by an anastomotic leak). Peripancreatic oedema can also be observed (b, white arrows)

release, due to systemic inflammatory response syndrome and pancreatic ischaemia, causing increased capillary permeability and the loss of intravascular oncotic pressure; however, this sign can also be identified in patients with pancreatitis [23-27, 32, 33].

\section{Splenic hypoperfusion}

The spleen is among the most vulnerable abdominal organs to hypotensive shock and often shows extremely decreased enhancement in early-phase CT (Figs. 2c-d). The hypoenhancement of the spleen and a reduction in 
the splenic volume are associated with severe hypoperfusion. The degree of hypoenhancement is believed to be directly related to hypoperfusion due to shock because the splenic artery has not autoregulatory mechanisms. Splenic hypoperfusion appears to be a useful predictor of poor prognosis among patients with systemic hypotension $[23-27,31]$.

\section{Liver hypoperfusion}

Hepatic enhancement is typically heterogeneous during hypotensive shock complex. A reduction in hepatic enhancement ( $25 \mathrm{HU}$ less than the spleen) is thought to be significant due to oedema (Fig. 4c) [23]. This CT manifestation is less common than other solid parenchymal abnormalities. False-positive interpretations could occur in patients with diffuse underlying liver disease, such as hepatic steatosis or liver congestion venous stasis [23-27]

\section{Abnormal renal enhancement}

Abnormal renal perfusion typically manifests as an increased and prolonged parenchymal enhancement (Fig. 7); however, focal and heterogeneous enhancement can also be observed. A fall in systolic pressure causes intense efferent glomerular arteriolar vasoconstriction, which drives glomerular filtration, leading to tubular stasis and the increased resorption of salt and water. Renal parenchymal enhancement is dependent on several factors, including cardiac output and scans timing relative to the injection of contrast agent and, thus, is a non-specific sign [23-27, 31]. However, kidney enhancement can vary depending on the severity of systemic hypotension. In some cases, unlike hyperenhancement, the decreased enhancement of the renal medulla can be observed in the venous phase, likely due to the impairment of contrast medium outflow from the renal cortex to the medulla, induced by acute renal tubular dysfunction and associated with poor prognosis (Figs. 4d, 8) [26].

\section{Abnormal adrenal enhancement (the adrenal stress)}

The bilateral hyperenhancement of the adrenal gland is more common in paediatric cases than in adults and can also present in combination with acute adrenal haemorrhage, which most commonly affects the right side unilaterally, with a homogeneous increase in the size of the gland and the associated suffusion of fat around the adrenal gland (Figs. 7a, 9). Bilateral adrenal hyperenhancement is the manifestation of adrenergic mechanisms that enhance the blood flow to the vital organs [23-27, 34, 35]. In the arterial phase, the central zone of the adrenal gland shows less intense enhancement than the peripheral zone or presents a mosaic appearance due to the heterogeneous enhancement of the central zone (Fig. 10). In both cases, in the venous phase, the whole adrenal gland is homogenously enhanced [36]. This sign highlights the central role played by the adrenal glands in mediating the sympathetic response to hypotensive shock and is associated with poor prognosis [23-27, 34, 35].

\section{Abnormal thyroid enhancement (shock thyroid)}

The thyroid gland, in the absence of direct damage, presents an increase sized, heterogeneous contrast hyperenhancement, similar to a multinodular gland, with the presence of low-density fluid surrounding the thyroid (5-10 HU) (Fig. 11). Thyroid shock is a minor finding associated with hypotensive shock complex in the absence of any known direct or indirect thyroid injury [23-27, 37, 38].
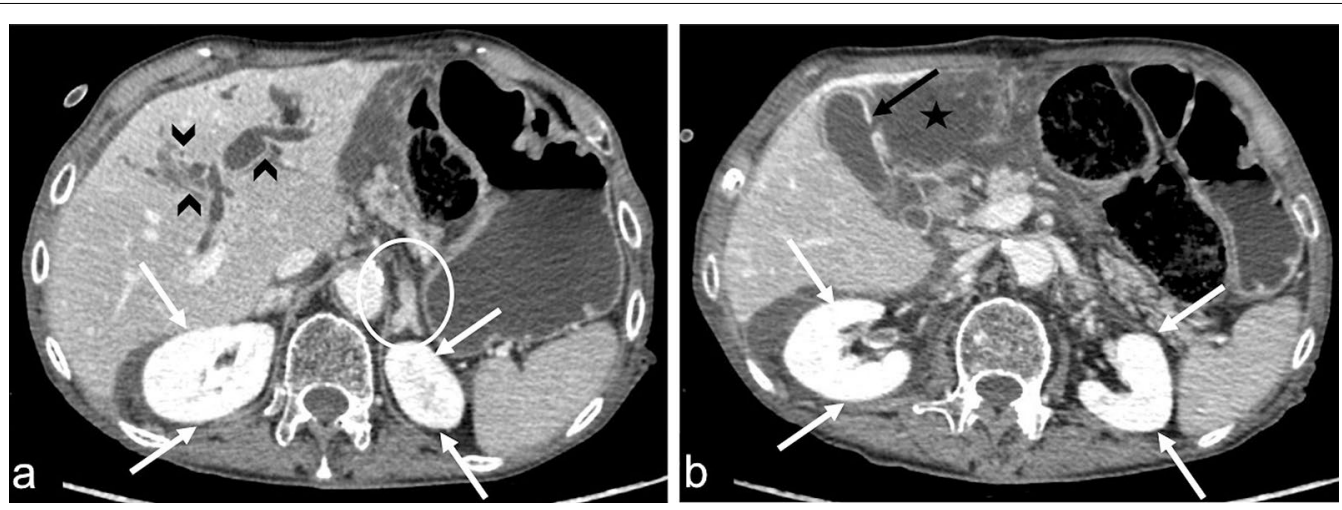

Fig. 7 Contrast-enhanced CT images in the portal venous phase, in the axial view, shows a 92-year-old male with sepsis of the biliary tract (qSOFA 3) characterised by segmental intrahepatic biliary duct dilatation (a, black arrowheads) and gallbladder leak (b, black arrow) with extrahepatic biloma (b, black star). Note the increased renal parenchymal enhancement bilaterally (a, b, white arrows) and the abnormal adrenal enhancement (a, white circle) 

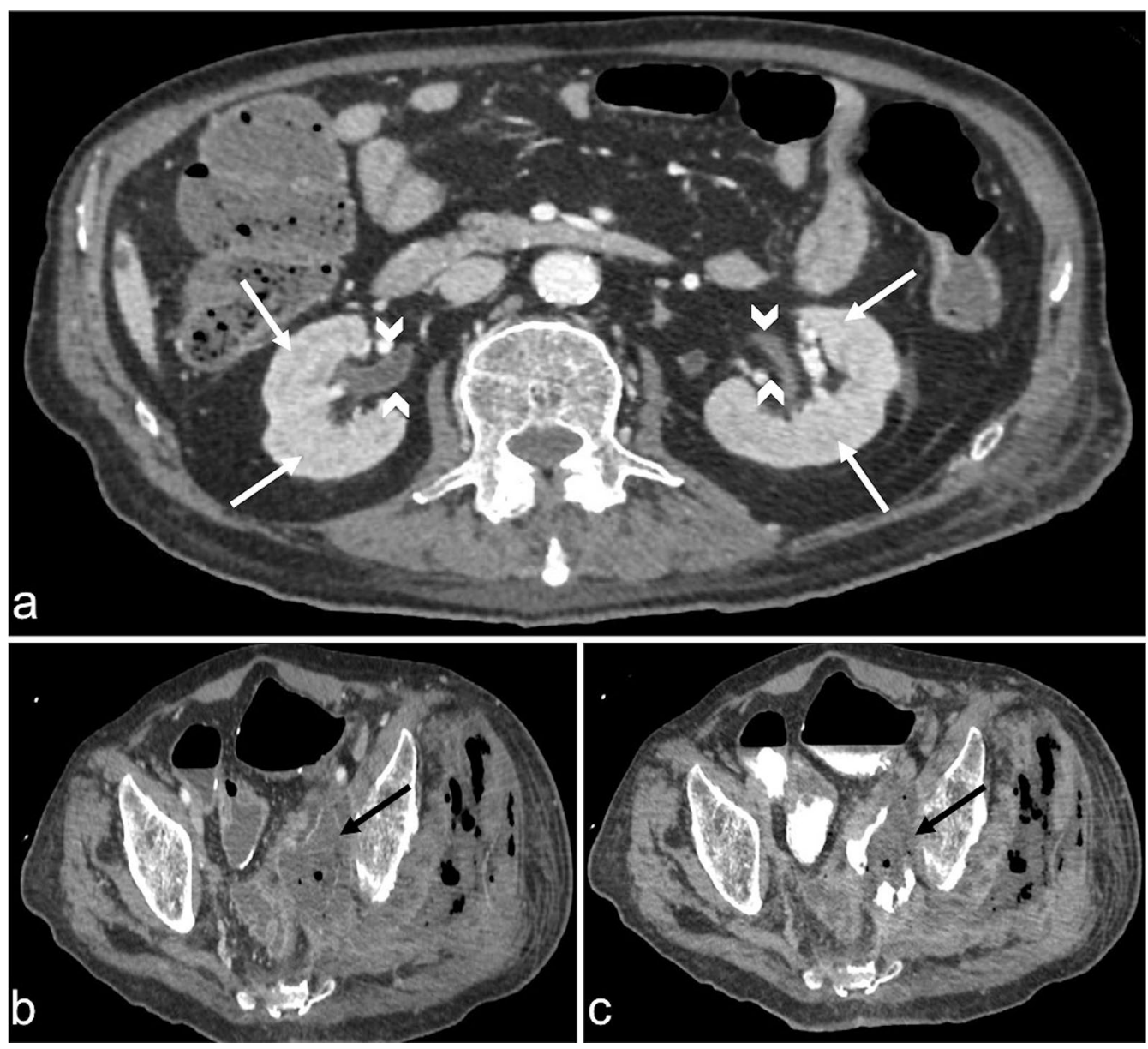

Fig. 8 Contrast-enhanced CT images in the portal venous phase ( $\mathbf{a}$ and $\mathbf{b})$ and the three-minute-delayed phase (c), showing a patient with septic shock (qSOFA 2) due to entero-neovesical fistula (b and c, black arrows) with pyelitis (a, arrowheads) after radical cystectomy. The decreased enhancement of the renal medulla (a, white arrows) in the venous phase was observed

\section{Other sign}

\section{Ascites}

The presence of ascites correlates with multiorgan dysfunction and is a non-specific sign [39].

\section{Conclusion}

In previously published studies, CT hypoperfusion complex has been almost exclusively focused on trauma-induced hypotensive shock and only few studies correlated these signs with prognosis. Some studies have suggested that flattering of IVC, shock bowel, impaired renal enhancement and splenic hypoperfusion are the most suggestive signs of hypoperfusion complex, strongly correlated with a poor prognosis although in a series of trauma-related hypovolemic shock [23-26, 31].
In contrast, only adrenal hyperenhancement has been correlated with a poor prognosis in septic shock [36]. Despite these contradictory reports, understanding these findings could prompt the development of alternative approaches for the early assessment and management of septic shock in the emergency setting [26]. Therefore, in addition to the application of CT for determining the underlying cause of the septic state, clinicians should be aware and be able to recognise the various CT findings that are suggestive of the hypotensive state. In this perspective, CT imaging represents a useful tool for a complete, rapid, and detailed diagnosis of clinically suspected septic shock, which can be used to improve patient outcomes. 


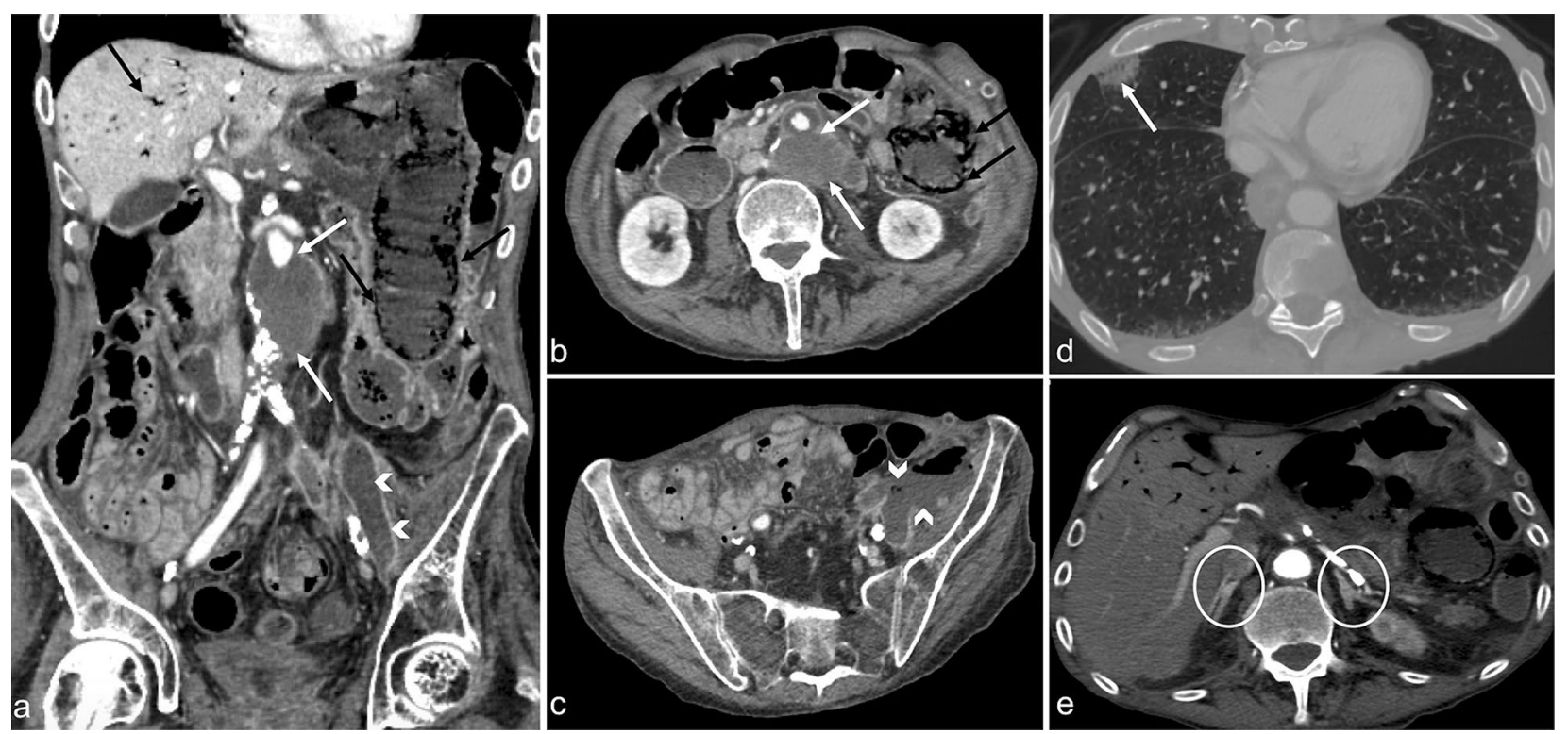

Fig. 9 Contrast-enhanced CT images in the arterial phase showing a 75-year-old male with sepsis (qSOFA 3) due to an infected aneurysm after aorto-basilic stent placement (a and $\mathbf{b}$, white arrows). Concomitant intestinal images reveal liver septic pneumatosis (a and $\mathbf{b}$, black arrows), stercoraceous collection in the left iliac fossa (c, white arrowheads), and septic emboli with pulmonary infarction (d, white arrows). The adrenal glands display hyperenhancement (e, white circles)
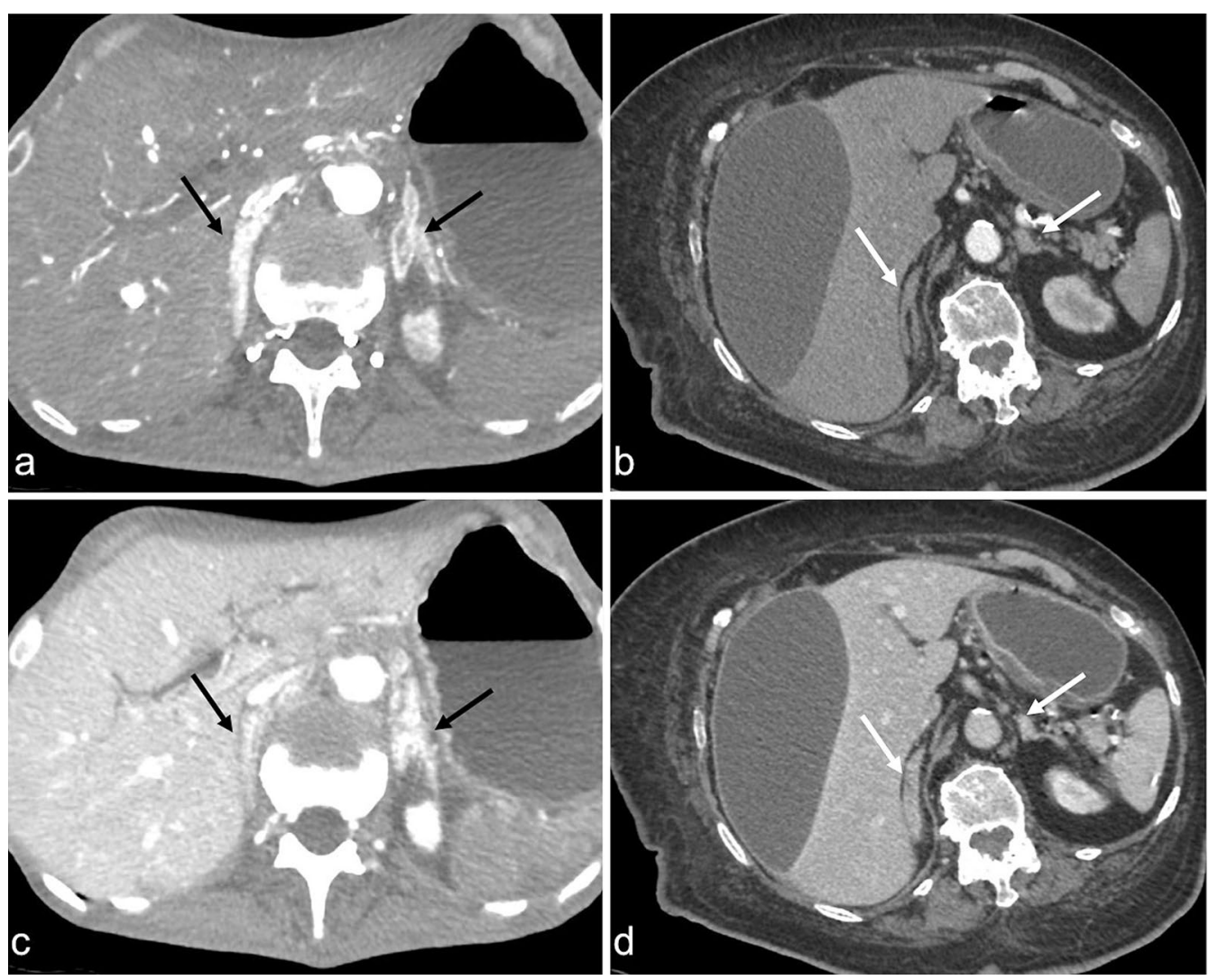

Fig. 10 Contrast-enhanced CT images in the arterial phase from two different patients with septic shock (a and $\mathbf{b})$ and the respective venous phases (c and $\mathbf{d}$ ). Different patterns of adrenal gland hyperenhancement can be observed, characterised in one patient by a central zone with less intense enhancement relative to the peripheral zone (a, black arrows) and in the other patient by a central zone with heterogeneous enhancement and a mosaic-like appearance (b, white arrows). In both cases, the venous phase shows a homogeneous enhancement (c, black arrows; $\mathbf{d}$, white arrows) 

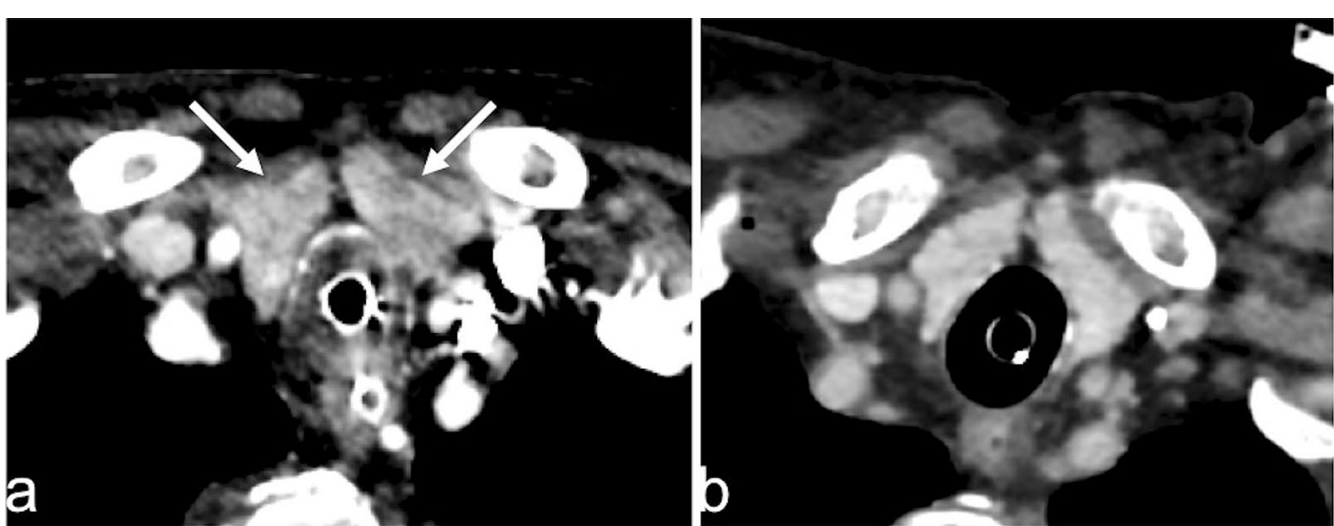

Fig. 11 Contrast-enhanced CT image in the portal venous phase showing an 89-year-old female with sepsis due to bowel wall perforation, secondary to colon cancer (qSOFA 3). An inhomogeneous enhancement of the thyroid gland was observed (a, arrow), in contrast with the previously homogeneous enhancement of the gland 4 days earlier (b)

\section{Abbreviations}

ARDS: Acute respiratory distress syndrome; CEUS: Contrast-enhancement ultrasound; CT: Computed tomography; DIC: Disseminated intravascular coagulation; ED: Emergency department; HU: Hounsfield unit; ICU: Intensive care unit; IV: Intravenous; IVC: Inferior vein cava; MAP: Mean arterial pressure; MIP: Maximum intensity projection; MPR: Multiplanar reformation; MRI: Magnetic resonance imaging; qSOFA: Quick sequential organ failure assessment; ROI: Region of interest; US: Ultrasound.

\section{Acknowledgements}

The authors would like to thank Dr Giovanni Palma for collecting the data.

\section{Authors' contributions}

MDS, DV, LG, and FI wrote the manuscript. MDS, FI, LB, FP, RR, AR, AB, GO, and $L R$ collected data and contributed to image collection. LR and MGDC made critical revisions to the manuscript with respect to important intellectual content. All authors critically revised the manuscript, approved the final version to be published, and agree to be accountable for all aspects of the work.

\section{Funding}

Not applicable.

\section{Availability of data and materials}

Data sharing is not applicable to this article as no datasets were generated or analysed during the current study.

\section{Declarations}

Ethics approval and consent to participate

Not applicable.

\section{Consent for publication}

Not applicable.

\section{Informed consent}

The patient's name has been removed from all images and cannot be recognized in any way.

\section{Competing interests}

The authors declare that they have no competing interests.

\section{Author details}

'Department of General and Emergency Radiology, "Antonio Cardarelli" Hospital, Antonio Cardarelli st 9, 80131 Naples, Italy. ${ }^{2}$ Department of Anesthesia and Resuscitation, "Antonio Cardarelli" Hospital, Naples, Italy. ${ }^{3}$ Department of Advanced Biomedical Sciences, "Federico II" University Hospital, Naples, Italy. ${ }^{4}$ Department of Vascular and Interventional Radiology, "Antonio Cardarelli" Hospital, Naples, Italy.
Received: 16 December 2020 Accepted: 5 May 2021

Published online: 05 June 2021

\section{References}

1. Singer M, Deutschman CS, Seymour CW et al (2016) The third international consensus definitions for sepsis and septic shock (Sepsis-3). JAMA 315(8):801-810. https://doi.org/10.1001/jama.2016.0287

2. Dellinger RP, Levy MM, Rhodes A et al (2013) Surviving Sepsis Campaign: International guidelines for management of severe sepsis and septic shock 2012. Intensive Care Med 39(2):165-228. https://doi.org/10.1007/ s00134-012-2769-8

3. Levy MM, Fink MP, Marshall JC et al (2001) SCCM/ESICM/ACCP/ATS/ SIS international sepsis definition conference. Intensive Care Med 29(4):530-538. https://doi.org/10.1007/s00134-003-1662-x

4. Shankar-Hari M, Phillips GS, Levy ML et al (2016) Developing a new definition and assessing new clinical criteria for septic shock: for the third international consensus definitions for sepsis and septic shock (Sepsis-3). JAMA 315(8):775-787. https://doi.org/10.1001/jama.2016.0289

5. Levy MM, Evans LE, Rhodes A (2018) The surviving sepsis campaign bundle: 2018 update. Intensive Care Med 44(6):925-928. https://doi.org/ 10.1007/s00134-018-5085-0

6. Dugar S, Choudhary C, Duggal A (2020) Sepsis and septic shock: guideline-based management. Clevel Clin J Med 87(1):53-64. https://doi. org/10.3949/ccjm.87a.18143

7. Vendemiato AV, von Nowakonski A, Marson FA, Levy CE (2015) Microbiological characteristics of sepsis in a university hospital. BMC Infect Dis 14(15):58. https://doi.org/10.1186/s12879-015-0798-y

8. Angus DC, van der Poll T (2013) Severe sepsis and septic shock. N Engl J Med 369(9):840-851. https://doi.org/10.1056/NEJMra1208623

9. Gruartmoner G, Mesquida J, Ince C (2017) Microcirculatory monitoring in septic patients: where do we stand? Med Intensiva 41(1):44-52. https:// doi.org/10.1016/j.medin.2016.11.011

10. Gauer RL (2013) Early recognition and management of sepsis in adults: the first six hours. Am Fam Physician 88(1):44-53

11. Rhee C, Zhang Z, Kadri SS et al (2019) CDC prevention epicenters program. Sepsis surveillance using adult sepsis events simplified eSOFA criteria versus sepsis-3 sequential organ failure assessment criteria. Crit Care Med 47(3):307-314. https://doi.org/10.1097/CCM.0000000000003521

12. Thompson K, Venkatesh B, Finfer S (2019) Sepsis and septic shock: current approaches to management. Intern Med J 49(2):160-170. https://doi.org/ 10.1111/imj.14199

13. Woodhead M, Aliyu S, Ashton C et al (2014) Guideline development group diagnosis and management of community and hospital acquired pneumonia in adults: summary of NICE guidance. BMJ 349:96722. https:// doi.org/10.1136/bmj.g6722 
14. Wagenlehner FM, Tandogdu Z, Bjerklund Johansen TE (2017) An update on classification and management of urosepsis. Curr Opin Urol 27(2):133-137. https://doi.org/10.1097/MOU.0000000000000364

15. Sartelli M, Catena F, Abu-Zidan FM et al (2017) Management of intraabdominal infections: recommendations by the WSES 2016 consensus conference. World J Emerg Surg 4(12):22. https://doi.org/10.1186/ s13017-017-0132-7

16. Adam EJ, Page JE (1991) Intra-abdominal sepsis: the role of radiology. Baillieres Clin Gastroenterol 5(3 Pt 1):587-609. https://doi.org/10.1016/ 0950-3528(91)90044-2

17. Creamer A, Keep J (2014) Imaging in severe sepsis and septic shock: is early radiological identification of occult sources of infection needed? Crit Care 18(Suppl 2):P12. https://doi.org/10.1186/cc14015

18. Di Serafino M, Vallone $G$ (2020) The role of point of care ultrasound in radiology department: update and prospective. A statement of Italian college ultrasound. Radiol Med. https://doi.org/10.1007/ s11547-020-01301-z

19. Haydar SA, Moore ET, Higgins GL 3rd et al (2012) Effect of bedside ultrasonography on the certainty of physician clinical decision making for septic patients in the emergency department. Ann Emerg Med 60(3):346-58.e4. https://doi.org/10.1016/j.annemergmed.2012.01.006

20. Guérin L, Vieillard-Baron A (2016) The use of ultrasound in caring for patients with sepsis. Clin Chest Med 37(2):299-307. https://doi.org/10. 1016/j.ccm.2016.01.005

21. Di Serafino M, Notaro M, Rea G, lacobellis F et al (2020) The lung ultrasound: facts or artifacts? In the era of COVID-19 outbreak. Radiol Med 125(8):738-753. https://doi.org/10.1007/s11547-020-01236-5

22. Pescatori LC, Brambati M, Messina C, Mauri G, Di Leo G et al (2018) Clinical impact of computed tomography in the emergency department in nontraumatic chest and abdominal conditions. Emerg Radiol 25(4):393-398. https://doi.org/10.1007/s10140-018-1592-0

23. Tarrant AM, Ryan MF, Hamilton PA, Benjaminov O (2008) A pictorial review of hypovolaemic shock in adults. Br J Radiol 81(963):252-257. https://doi. org/10.1259/bjr/40962054

24. Ames JT, Federle MP (2009) CT hypotension complex (shock bowel) is not always due to traumatic hypovolemic shock. AJR Am J Roentgenol 192(5):W230-W235. https://doi.org/10.2214/AJR.08.1474

25. Higashi H, Kanki A, Watanabe S et al (2014) Traumatic hypovolemic shock revisited: the spectrum of contrast-enhanced abdominal computed tomography findings and clinical implications for its management. Jpn J Radiol 32:579-584. https://doi.org/10.1007/s11604-014-0354-5

26. Wang J, Liang T, Louis L, Nicolaou S, McLaughlin PD (2013) Hypovolemic shock complex in the trauma setting: a pictorial review. Can Assoc Radiol J 64(2):156-163. https://doi.org/10.1016/j.carj.2013.03.002

27. Shin MS, Berland LL, Ho KJ (1990) Small aorta: CT detection and clinical significance. J Comput Assist Tomogr 14(1):102-103
28. Hara H, Babyn PS, Bourgeois D (1992) Significance of bowel wall enhancement on CT following blunt abdominal trauma in childhood. J Comput Assist Tomogr 16(1):94-98. https://doi.org/10.1097/00004728199201000-00017

29. Macari M, Balthazar EJ (2001) CT of bowel wall thickening: significance and pitfalls of interpretation. AJR Am J Roentgenol 176(5):1105-1116. https://doi.org/10.2214/ajr.176.5.1761105

30. Sugi MD, Menias CO, Lubner MG et al (2018) CT findings of acute smallbowel entities. Radiographics 38(5):1352-1369. https://doi.org/10.1148/ rg.2018170148

31. Kanki A, Ito K, Tamada T et al (2011) Dynamic contrast-enhanced CT of the abdomen to predict clinical prognosis in patients with hypovolemic shock. AJR Am J Roentgenol 197(6):W980-W984. https://doi.org/10.2214/ AJR.10.5736

32. lacobellis F, Laccetti E, Tamburrini S, Altiero M, laselli F, Di Serafino M et al (2019) Role of multidetector computed tomography in the assessment of pancreatic injuries after blunt trauma: a multicenter experience. Gland Surg 8(2):184-196. https://doi.org/10.21037/gs.2019.02.02

33. Ryan MF, Hamilton PA, Sarrazin J et al (2005) The halo sign and peripancreatic fluid: useful CT signs of hypovolaemic shock complex in adults. Clin Radiol 60(5):599-607. https://doi.org/10.1016/j.crad.2004.02.012

34. Venkatanarasimha N, Roobottom C (2010) Intense adrenal enhancement: a feature of hypoperfusion complex. AJR Am J Roentgenol 195(1):W82. https://doi.org/10.2214/AJR.09.3829

35. Di Serafino M, Severino R, Coppola V, Gioioso M et al (2017) Nontraumatic adrenal hemorrhage: the adrenal stress. Radiol Case Rep 12(3):483-487. https://doi.org/10.1016/j.radcr.2017.03.020

36. Peng $Y$, Xie Q, Wang $\mathrm{H}$ et al (2019) The hollow adrenal gland sign: $\mathrm{a}$ newly described enhancing pattern of the adrenal gland on dualphase contrast-enhanced CT for predicting the prognosis of patients with septic shock. Eur Radiol 29:5378-5385. https://doi.org/10.1007/ s00330-019-06172-1

37. Han DH, Ha EJ, Sun JS, Jung SL (2017) Remarkable CT features of shock thyroid in traumatic and non-traumatic patients. Emerg Radiol 24(3):319324. https://doi.org/10.1007/s10140-016-1475-1

38. Brochert A, Rafoth JB (2006) Shock thyroid: a new manifestation of the hypovolemic shock complex in trauma patients. J Comput Assist Tomogr 30(2):310-312. https://doi.org/10.1097/00004728-200603000-00028

39. Rossaint J, Zarbock A (2015) Pathogenesis of multiple organ failure in sepsis. Crit Rev Immunol 35(4):277-291. https://doi.org/10.1615/critrevimm unol.2015015461

\section{Publisher's Note}

Springer Nature remains neutral with regard to jurisdictional claims in published maps and institutional affiliations.

\section{Submit your manuscript to a SpringerOpen ${ }^{\circ}$ journal and benefit from:}

- Convenient online submission

- Rigorous peer review

- Open access: articles freely available online

- High visibility within the field

Retaining the copyright to your article

Submit your next manuscript at $\boldsymbol{\nabla}$ springeropen.com 